\title{
Errata: Hardware acceleration of a Monte Carlo simulation for photodynamic therapy treatment planning
}

\author{
William Chun Yip Lo \\ University of Toronto \\ Department of Medical Biophysics \\ Rm. 8-324 \\ 610 University Avenue \\ Toronto, Ontario M5G 2M9 Canada
}

\author{
Keith Redmond \\ Jason Luu \\ Paul Chow \\ Jonathan Rose \\ University of Toronto \\ The Edward S. Rogers Sr. Department of Electrical \\ and Computer Engineering \\ 10 King's College Road \\ Toronto, Ontario M5S 3G4
}

\section{Lothar Lilge}

Princess Margaret Hospital

Ontario Cancer Institute

University of Toronto

Department of Medical Biophysics

Rm. 7-416

610 University Avenue

Toronto, Ontario M5G 2M9 Canada

[DOI: $10.1117 / 1.3125246]$

This article [J. Biomed. Opt. 14, 014019 (January/February 2009)] was originally published online on 27 February 2009 with errors. All online versions of the article were corrected on 17 March 2009. A listing of the corrections follows here:

1. The title was changed from "Hardware acceleration of a Monte Carlo simulation for photodynamic treatment planning" to "Hardware acceleration of a Monte Carlo simulation for photodynamic therapy treatment planning."

2. In the first paragraph of Section 3.2, the second to the last sentence was changed from "Structural design requires that a hardware design specifying..." to Structural design requires a hardware designer to specify..."

3. Equation (1) was changed from

$$
\mu_{x}^{\prime}=\frac{\sin \theta\left(\mu_{x} \mu_{z} \cos \psi-\mu_{y} \sin \psi\right)}{\sqrt{1-\mu_{z}^{2}}+\mu_{x} \cos \theta} .
$$

to

$$
\mu_{x}^{\prime}=\frac{\sin \theta\left(\mu_{x} \mu_{z} \cos \psi-\mu_{y} \sin \psi\right)}{\sqrt{1-\mu_{z}^{2}}}+\mu_{x} \cos \theta .
$$

4. The reference mentioned in the paragraph above Eq. (1) on page 4 was changed from 16 to 17 .
5. On page 5, last paragraph of Section 4.1, the sentence "This is possible because the result from only two of the three modules is used..." has been changed to "This is possible because the result from only either modules $4 \mathrm{~b}$ and $4 \mathrm{c}$ or module $4 \mathrm{a}$ alone is used..."

6. Under the column "Clock Speed" in Tables 3(a) and 3(b), row "TM-4," the value was changed from $40 \mathrm{MHz}$ to $41 \mathrm{MHz}$. 